\title{
Spin-orbit-coupled Bose-Einstein condensates held under toroidal trap
}

\author{
Xiao-Fei Zhang, ${ }^{1,2}$ Masaya Kato, ${ }^{1}$ Wei Han, ${ }^{2}$ Shou-Gang Zhang, ${ }^{2}$ and Hiroki Saito ${ }^{1}$ \\ ${ }^{1}$ Department of Engineering Science, University of Electro-Communications, Tokyo 182-8585, Japan \\ ${ }^{2}$ Key Laboratory of Time and Frequency Primary Standards, \\ National Time Service Center, Chinese Academy of Sciences, Xi'an 710600, China
}

(Dated: June 8, 2021)

\begin{abstract}
We study a quasispin-1/2 Bose-Einstein condensate with synthetically generated spin-orbit coupling in a toroidal trap, and show that the system has a rich variety of ground and metastable states. As the central hole region increases, i.e., the potential changes from harmonic-like to ring-like, the condensate exhibits a variety of structures, such as triangular stripes, flower-petal patterns, and counter-circling states. We also show that the rotating systems have exotic vortex configurations. In the limit of a quasi-one dimensional ring, the quantum many-body ground state is obtained, which is found to be the fragmented condensate.

PACS numbers: 03.75.Lm, 03.75.Mn, 67.85.Bc, 67.85.Fg
\end{abstract}

\section{INTRODUCTION}

The engineering of synthetic gauge field and spin-orbit coupling (SOC) in neutral atomic gases has recently attracted major attention both theoretically and experimentally [1 10]. In condensed matter physics, the SOC plays an important role for the emergence of many exotic quantum phenomena [11, 12]. The creation of SOC in spinor Bose-Einstein condensates (BECs) not only offers us a new platform to simulate the response of charged particles to external electromagnetic field, but also opens up an entirely new paradigm for studying strong correlations of quantum many-body systems, which enables quantum simulations of condensed matter phenomena because of the high controllability of the system [13 16]. Very recently, another type of SOC, namely, the spin and orbital-angular-momentum coupling, has also been proposed [17 19].

For a homogeneous spin-orbit (SO) coupled condensate, the mean-field ground state favors either a plane wave or a striped wave depending on the ratio between inter- and intra-component interactions [20, 21]. The presence of the trapping potential modifies this situation and leads to a rich ground-state physics 22 28]. In the presence of a two-dimensional (2D) harmonic trap, a complex phase diagram of Rashba SO-coupled Bose gases was observed, in which there are two classes of phases and several subphases 23. In addition, SO coupled BECs subject to rotation have been studied, which exhibit a rich variety of the ground-state phases and vortex configurations depending on the strength of SOC and rotation frequency [29 33].

Bosonic gases loaded in a toroidal trap have attracted considerable interest [34 40], where such a trapping potential can be realized by a blue-detuned laser beam to make a repulsive potential barrier in the middle of a harmonic magnetic trap [41]. The toroidal trap provides us an ideal platform to study fascinating properties of a superfluid, such as persistent flow [42 44] and symmetry breaking localization [45, 46]. Now the point is that, in the presence of a toroidal trap, we are inquisitive about whether such a potential can essentially change the properties of a SO coupled BEC with or without rotation, which is what we attempt to do in this work.

The paper is organized as follows. In Sec. II we formulate the theoretical model describing the SO coupled BECs held under toroidal trap. Various ground and metastable states generated by the effects of the SOC and toroidal potential are investigated using the meanfield theory in Sec. III. The quantum many-body ground state is studied in the limit of quasi-1D ring in Sec. IV. The main results of the paper are summarized in Sec. V.

\section{MODEL}

We consider a two-component BEC with Rashba SOC confined in a quasi-2D toroidal trap on the $x-y$ plane. The second-quantized Hamiltonian of the system is given by $\hat{\mathcal{H}}=\hat{\mathcal{H}}_{0}+\hat{\mathcal{H}}_{\text {int }}$, where

$$
\begin{aligned}
\hat{\mathcal{H}}_{0} & =\int d \mathbf{r} \hat{\boldsymbol{\psi}}^{\dagger}\left[-\frac{\hbar^{2} \nabla^{2}}{2 M}+\mathcal{V}_{s o}+V(r)-\Omega \hat{L}_{z}\right] \hat{\boldsymbol{\psi}}, \\
\hat{\mathcal{H}}_{\mathrm{int}} & =\int d \mathbf{r}\left(\frac{\mathrm{g}_{\uparrow \uparrow}}{2} \hat{\psi}_{\uparrow}^{\dagger 2} \hat{\psi}_{\uparrow}^{2}+\frac{\mathrm{g}_{\downarrow \downarrow}}{2} \hat{\psi}_{\downarrow}^{\dagger 2} \hat{\psi}_{\downarrow}^{2}+\mathrm{g}_{\uparrow \downarrow} \hat{\psi}_{\uparrow}^{\dagger} \hat{\psi}_{\downarrow}^{\dagger} \hat{\psi}_{\downarrow} \hat{\psi}_{\uparrow}\right),
\end{aligned}
$$

where $\hat{\boldsymbol{\psi}}=\left(\hat{\psi}_{\uparrow}, \hat{\psi}_{\downarrow}\right)^{T}$ denotes the field operator of the atom with pseudospin state $\uparrow, \downarrow$, and $M$ is the atomic mass. The Rashba SOC is $\mathcal{V}_{\mathrm{so}}=-i \kappa\left(\sigma_{x} \partial_{x}+\sigma_{y} \partial_{y}\right)$ with $\sigma_{x, y}$ being the Pauli matrices and $\kappa$ is the strength of the SOC. $\Omega$ is the rotation frequency and $\hat{L}_{z}$ is the $z$ component of the orbital angular momentum operator, where we assume that the "SOC lasers" are also rotated with the system to simplify the problem [30]. Here we further assume that the two intracomponent interaction parameters are the same, $g_{\uparrow \uparrow}=g_{\downarrow \downarrow} \equiv$ g. When the quasi$2 \mathrm{D}$ system is realized by a tight harmonic potential with frequency $\omega_{z}$, the effective interaction parameters are given by $\mathrm{g}=\sqrt{8 \pi} \hbar^{2} a /\left(M a_{z}\right)$ and $\mathrm{g}_{\uparrow \downarrow}=\sqrt{8 \pi} \hbar^{2} a_{\uparrow \downarrow} /\left(M a_{z}\right)$, 
where $a$ and $a_{\uparrow \downarrow}$ are the corresponding $s$-wave scattering lengths and $a_{z}=\sqrt{\hbar /\left(M \omega_{z}\right)}$.

The trapping potential considered here is a toroidal trap, which reads

$$
V(r)=\frac{1}{2} M \omega_{\perp}^{2} r^{2}+V_{0} e^{-2 r^{2} / \sigma_{0}^{2}},
$$

where $\omega_{\perp}$ is the radial trap frequency of the harmonic potential, $r^{2}=x^{2}+y^{2}$, and $V_{0}$ and $\sigma_{0}$ are proportional to the intensity and beam waist of the optical plug. The bottom of the potential in Eq. (2) is located at

$$
R=\frac{\sigma_{0}}{\sqrt{2}} \sqrt{\ln \frac{4 V_{0}}{M \omega_{\perp}^{2} \sigma_{0}^{2}}} .
$$

Expanding Eq. (2) around $r=R$ and neglecting the third and higher orders of $r-R$, we obtain

$$
V_{r}(r)=\frac{M \omega_{0}^{2}}{2}(r-R)^{2},
$$

where $\omega_{0}=2 \omega_{\perp} R / \sigma_{0}$ and a constant is omitted. This approximation is valid, when $\hbar \omega_{0}$ is much larger than the characteristic energy of the system.

\section{MEAN-FIELD ANALYSIS}

\section{A. Ground states}

We implement the mean-field approximation by replacing the field-operators $\hat{\psi}_{\uparrow, \downarrow}$ with the macroscopic wave functions $\psi_{\uparrow, \downarrow}$ in Eq. (1), which gives the GrossPitaevskii energy functional. We numerically minimize it by using the imaginary time evolution method and obtain the ground-state wave function. We work in dimensionless units by scaling with the appropriate factors of the harmonic trap energy $\hbar \omega_{\perp}$ and the harmonic trap length $\sqrt{\hbar /\left(M \omega_{\perp}\right)}$. The trapping potential in Eq. (2) can be rewritten as $V_{r}(r)=r^{2} / 2+A e^{-r^{2} / l^{2}}$ with $l=\sqrt{M \omega_{\perp} \sigma_{0}^{2} /(2 \hbar)}$ and $A=V_{0} /\left(\hbar \omega_{\perp}\right)$. The trapping potential in Eq. (4) is $V(r)=\tilde{\omega}_{0}^{2}(r-R)^{2} / 2$ with $\tilde{\omega}_{0}=\omega_{0} / \omega_{\perp}$.

Figure 1 shows the ground-state density profiles of the system for $g<g_{\uparrow \downarrow}$, i.e., the two components are immiscible. For small $l$ and $\kappa$, the system exhibits the radial phase separation, where one component is surrounded by the other one, as shown in Fig. 1(a). If the SOC is absent and the ratio $\int\left|\psi_{\uparrow}\right|^{2} d \mathbf{r} / \int\left|\psi_{\downarrow}\right|^{2} d \mathbf{r}$ is not fixed, the ground state is occupied only by one component for the immiscible case. The two thin rings of $n_{\downarrow}$ in Fig. 1(a) is therefore the effect of the SOC. For strong SOC, the rotational symmetry is broken and the ground state shows modified stripe phase, as shown in Figs. 1(b) and 1(c), which are similar to those predicted for homogeneous condensates except for the central hole region [20, 22]. Near the central hole, the stripe is bent, and it seems that the stripe

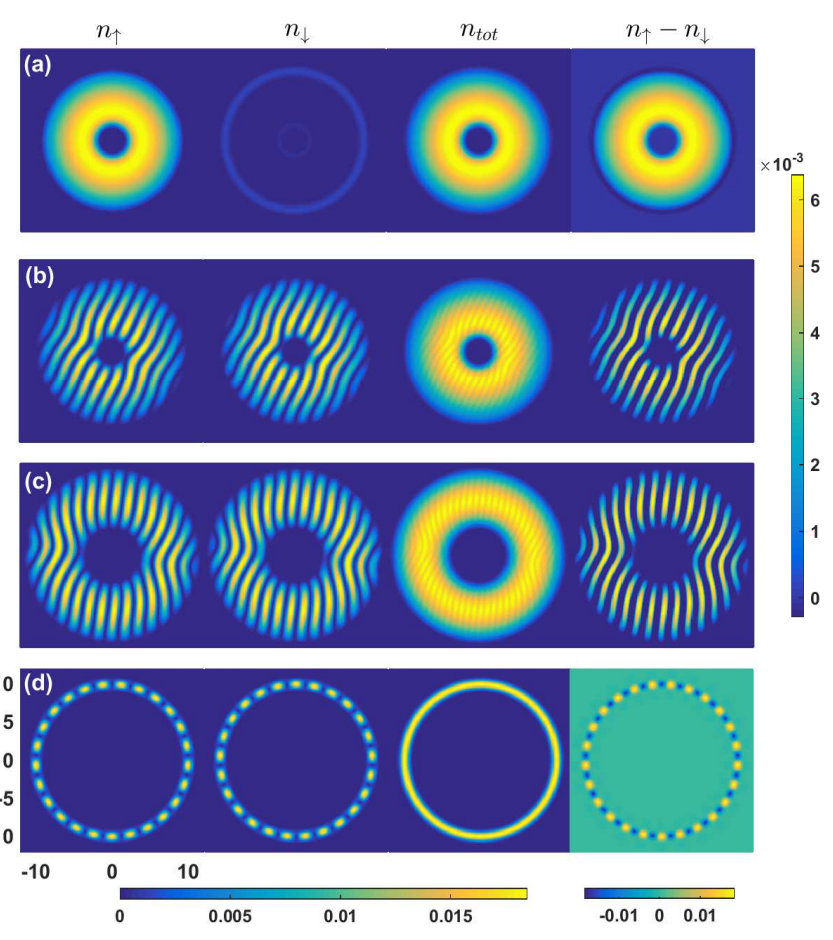

FIG. 1: (Color online) Examples of ground-state density profiles for the immiscible case. From left to right: densities of up- and down-components $n_{\uparrow}$ and $n_{\downarrow}$, total density $n_{\uparrow}+n_{\downarrow}$, and the density difference $n_{\uparrow}-n_{\downarrow}$. Panels (a) and (b) are for $l=2$, but for $\kappa=1$ and 2, respectively; while (c) is for $l=5$ and $\kappa=2$. Other parameters are given as $\mathrm{g}=6000$, $\mathrm{g}_{\uparrow \downarrow}=8000$, and $A=100$. (d) Example of density profile of the system in a one-dimensional ring trap of Eq. (4) with $\mathrm{g}=100, \mathrm{~g}_{\uparrow \downarrow}=120, \tilde{\omega}_{0}=\sqrt{10}, R=10$, and $\kappa=1$.

tends to be perpendicular to the perimeter of the central hole. In the limit of very tight and narrow annulus, this tendency becomes significant; the ground-state density distribution of the system shows alternately arranged stripe pattern along the ring, as shown in Fig. 1(d). The number of stripes increases with the strength of the SOC.

Figure 2 shows the miscible cases with $g>g_{\uparrow \downarrow}$. When the central hole is small, the ground state is similar to the plane wave [20], where all the bosons are condensed into a single plane-wave state, and the direction of the plane wave is chosen in the $x-y$ plane breaking the rotational symmetry. As the central hole is increased, the effect of the toroidal potential emerges; the direction of the flow tends to be azimuthal, as shown in Fig. 2(a). As a result, the flow pattern becomes counter-circling. For a larger $l$ (close to the ring limit), this tendency becomes significant and the flow becomes one-way circulation, as shown in Fig. 2(b). Thus, the direction of the mass flow in the ground state changes with the shape of the toroidal trap. 

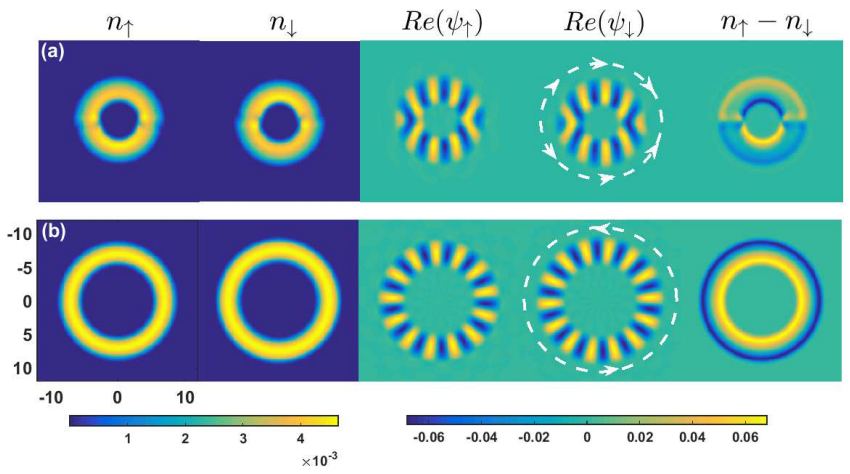

FIG. 2: (Color online) Typical examples of the ground states for the miscible case. From left to right: densities of upand down- components $n_{\uparrow}$ and $n_{\downarrow}$, the real parts $\operatorname{Re}\left(\psi_{\uparrow}\right)$ and $\operatorname{Re}\left(\psi_{\downarrow}\right)$, and the density difference $n_{\uparrow}-n_{\downarrow}$. The parameters are given as $\mathrm{g}=1000, \mathrm{~g}_{\uparrow \downarrow}=800, A=100, \kappa=2$, and $l=2$ in (a) and $l=5$ in (b). The direction of the mass flow is marked by the dashed-line arrows.

\section{B. Variational analysis}

The results in Figs. 1 and 2 indicate that the direction of the wave-number vector tends to be azimuthal in a toroidal trap, and this tendency is more significant for a tighter toroidal confinement. To understand this result, we employ the Gaussian variational method. For simplicity, we assume a potential $V=x^{2} / 2$, and show that the wave-number vector becomes perpendicular to the confinement, i.e., in the $y$ direction. For the miscible case, the variational wave functions are assumed to be

$$
\begin{aligned}
& \psi_{\uparrow}=\frac{1}{\sqrt{2}} \frac{1}{\pi^{1 / 4} \sigma^{1 / 2}} e^{-\frac{(x+X)^{2}}{2 \sigma^{2}}} e^{i \mathbf{k} \cdot \mathbf{r}}, \\
& \psi_{\downarrow}=\frac{1}{\sqrt{2}} \frac{-e^{i \phi_{\mathbf{k}}}}{\pi^{1 / 4} \sigma^{1 / 2}} e^{-\frac{(x-X)^{2}}{2 \sigma^{2}}} e^{i \mathbf{k} \cdot \mathbf{r}},
\end{aligned}
$$

where $\sigma, X$, and $\mathbf{k}$ are variational parameters and $\phi_{\mathbf{k}}$ is the angle between $\mathbf{k}$ and the $x$ axis. The shift $X$ in Eq. (5) is justified by the numerical result shown in the last column of Fig. 2, where $\left|\psi_{\uparrow}\right|^{2}$ and $\left|\psi_{\downarrow}\right|^{2}$ are shifted in the opposite directions perpendicular to the direction of confinement. Substituting Eq. (5) into the Gross-Pitaevskii energy functional, we obtain the variational energy as

$$
\begin{aligned}
E= & \frac{1}{4 \sigma^{2}}+\frac{k^{2}}{2}+\frac{\sigma^{2}}{4}+\frac{X^{2}}{2}-\kappa\left(k+\frac{k_{y}}{k} \frac{X}{\sigma^{2}}\right) e^{-\frac{X^{2}}{\sigma^{2}}} \\
& +\frac{1}{4 \sqrt{2 \pi} \sigma}\left(\mathrm{g}+\mathrm{g}_{\uparrow \downarrow} e^{-\frac{X^{2}}{\sigma^{2}}}\right) .
\end{aligned}
$$

We note that this energy is independent of the direction of $\mathbf{k}$, if $X=0$. Assuming $X \ll \sigma$, we find that $k_{x}=0$, $k_{y} \simeq \pm \kappa$, and $X \simeq \pm \kappa / \sigma^{2}$ minimize $E$. The energy of the state with $\mathbf{k}$ in the $y$ direction is smaller than that with $\mathbf{k}$ in the $x$ direction by $\simeq \kappa^{2} /\left(2 \sigma_{0}^{4}\right)$. Thus, in the miscible case, the ground state tends to have momentum perpendicular to the direction of confinement.
For the immiscible case, the variational wave function is assumed to be

$$
\begin{aligned}
& \psi_{\uparrow}=\frac{1}{2 \pi^{1 / 4} \sigma^{1 / 2}}\left[e^{-\frac{(x-X)^{2}}{2 \sigma^{2}}} e^{i \mathbf{k} \cdot \mathbf{r}}+e^{-\frac{(x+X)^{2}}{2 \sigma^{2}}} e^{-i \mathbf{k} \cdot \mathbf{r}}\right], \\
& \psi_{\downarrow}=\frac{-e^{i \phi_{\mathbf{k}}}}{2 \pi^{1 / 4} \sigma^{1 / 2}}\left[e^{-\frac{(x+X)^{2}}{2 \sigma^{2}}} e^{i \mathbf{k} \cdot \mathbf{r}}-e^{-\frac{(x-X)^{2}}{2 \sigma^{2}}} e^{-i \mathbf{k} \cdot \mathbf{r}}\right],
\end{aligned}
$$

which reduces to the usual stripe state for $X=0$. With the same procedure as above (see Appendix), we find that the energy is minimized by $k_{x}=0, k_{y} \simeq \pm \kappa$, and $X \simeq$ $\pm \kappa / \sigma^{2}$, which are the same as in the miscible case. Thus, in both miscible and immiscible cases, the direction of the wave-number vector $\mathbf{k}$ tends to be perpendicular to the direction of confinement, which explains the behaviors in Figs. 1 and 2, i.e., the wave-number vector tends to be azimuthal in a toroidal trap.

\section{Various metastable states}

There are various metastable states which have almost the same energy as the ground state, as shown in Fig. 3 . To trigger these various pattern formations, we start from different initial states in the imaginary-time propagation.

Figures 3(a) and 3(b) show two typical density distributions and its associated real part of the wave functions for the miscible case with the same parameter set, where the direction of the mass flow is marked by the dashedline arrows. It is clearly seen that different types of the mass flow can be triggered by a proper choice of the initial state.

Figures 3(c) and 3(d) show two different types of stationary states for the immiscible case with the same parameter set. The density distributions of these metastable states show flower-petal stripe patterns, which are quite different from the usual stripe phase. Interestingly, with a further increase in SOC, the stripe shows triangular structure, as shown in Fig. 3(e) for $\kappa=2$. To get a deeper physical insight into the origin of this state, it is useful to look at its momentum distribution, which is presented in the right panel of Fig. 3(e). One can see that six maxima at angles $\varphi=\pi / 3$ appear and form a ringlike momentum distribution. This is in a sense reminiscent of the harmonically-trapped case, where a lattice phase emerges as the ground state [22]. In the present case, large $l$ leads to a large radius of the toroidal trap and a large central hole, and the behavior of the system is intermediate between the harmonic potential and one-dimensional (1D) ring. As a result, the density distribution of the system shows the triangular stripe phase, where main region of the toroidal trap is occupied by the straight stripes and their vertices with an equilateral triangle shape by curved stripes. 


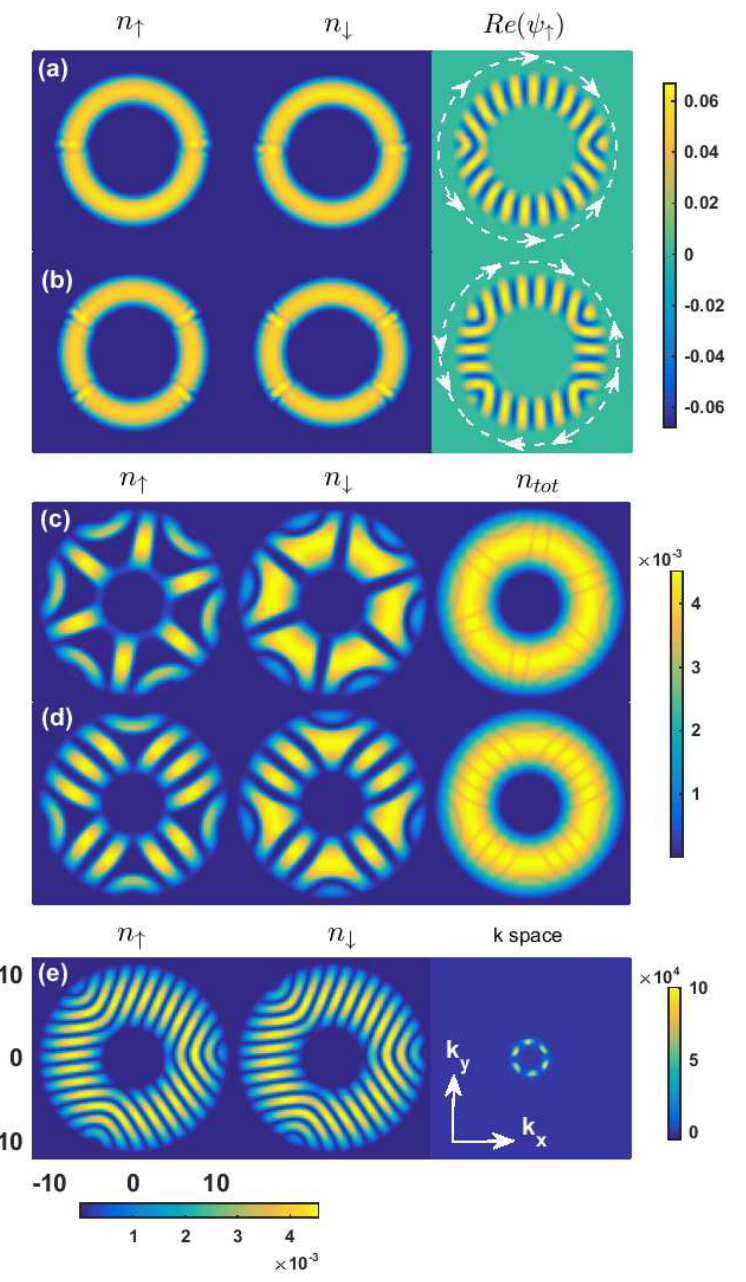

FIG. 3: (Color online) Typical stationary states of the system. (a)-(b) Two different types of density and flow patterns for the miscible case with $\mathrm{g}=1000, \mathrm{~g}_{\uparrow \downarrow}=800, A=100, l=5$, and $\kappa=3$, where the direction of the mass flow is marked by the dashed-line arrows. (c)-(d) Two different types of density profiles for the immiscible case with $\mathrm{g}=6000, \mathrm{~g}_{\uparrow \downarrow}=8000$, $A=100, l=5$, and $\kappa=1.25$; while (e) density and momentum distributions for $\kappa=2$.

\section{Rotational properties}

One of the interesting aspects of the toroidal trap is its rotational properties, which manifest themselves as the presence of quantized vortices and its related vortex lattices [47, 48]. The typical vortex structures of a harmonically-trapped BECs with SOC and rotation have been well studied, showing various exotic vortex lattices by varying the strength of SOC and rotation frequency [29 31, 49].

Our numerical results show that when the rotation frequency $\Omega$ is small, vortices are distributed on a circle and the number of vortices along the circle increases with $\Omega$, as shown in Figs. 4(a) and 4(b). With an increase in the rotation frequency, we find that not only the number of vortices along a single circle but also the number of cir-
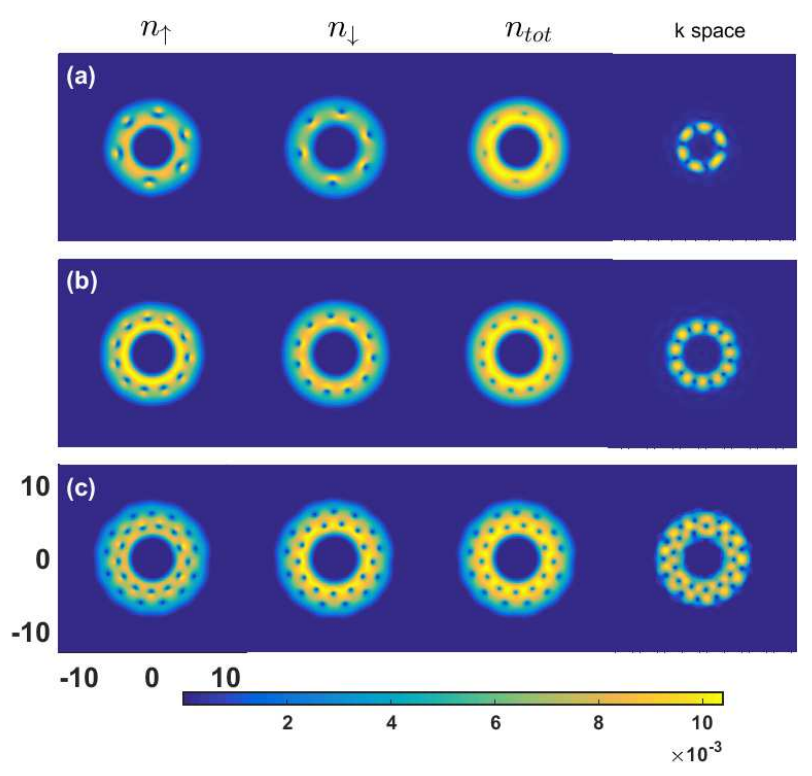

FIG. 4: (Color online) Ground states of the rotating system for $l=2$, and for rotation frequencies $\Omega=0.4,0.6$, and 0.8 , corresponding to (a), (b), and (c), respectively. From left to right: Density of up-component, density of down-component, total density, and $k$-space density of the spin-up component. Other parameters are given as $\mathrm{g}=6000, \mathrm{~g}_{\uparrow \downarrow}=8000, \kappa=1.0$, and $A=100$.

cles increases, as shown in Fig. 4(c). For fixed $l$ and $\Omega$, either the number of vortices on each circle or the number of vortex circles increases with the strength of SOC, which is easily explained by the fact that the SOC can help vortex nucleation. Interestingly, the momentumspace densities have similar structures to the real-space densities, as shown in the last column of Fig. 4. There are vortices also in the momentum space and the number of vortices in the momentum space is equal to that in real space.

\section{QUANTUM MANY-BODY ANALYSIS}

We now consider a quasi-1D ring potential, in which $\omega_{0}$ in Eq. (4) is so large that $\sqrt{\hbar /\left(M \omega_{0}\right)} \ll R$ and $\hbar \omega_{0}$ is much larger than any other energy scales. In this case, the atoms are confined to $r \simeq R$. The radial wave function can be approximated by the ground state of

$$
\left[-\frac{1}{2}\left(\frac{\partial^{2}}{\partial r^{2}}+\frac{1}{r} \frac{\partial}{\partial r}\right)+V_{r}(r)\right] f(r)=E_{0} f(r)
$$

where $\int_{0}^{\infty} f^{2}(r) r d r=1$. One can show $\int_{0}^{\infty} f^{2}(r) d r \simeq$ $1 / R$ and $\int_{0}^{\infty} f(r) f^{\prime}(r) r d r \simeq-1 /(2 R)$. Dividing the field operator into the radial and azimuthal parts as $\hat{\psi}_{\uparrow, \downarrow}(\mathbf{r})=$ 
$f(r) \hat{\phi}_{\uparrow, \downarrow}(\theta)$, the Hamiltonian $\hat{\mathcal{H}}$ in Eq. (1) reduces to

$$
\begin{aligned}
& \hat{\mathcal{H}}=\int_{0}^{2 \pi} d \theta\left\{\hat{\phi}^{\dagger}\left(E_{0}-\frac{1}{2 R^{2}} \frac{\partial^{2}}{\partial r^{2}}+i \Omega \frac{\partial}{\partial \theta}\right) \hat{\phi}\right. \\
& +\frac{i \kappa}{R}\left[e^{-i \theta} \hat{\phi}_{\uparrow}^{\dagger}\left(\frac{1}{2}+i \frac{\partial}{\partial \theta}\right) \hat{\phi}_{\downarrow}+e^{i \theta} \hat{\phi}_{\downarrow}^{\dagger}\left(\frac{1}{2}-i \frac{\partial}{\partial \theta}\right) \hat{\phi}_{\uparrow}\right] \\
& \left.+\frac{\mathrm{g} C}{2}\left(\hat{\phi}_{\uparrow}^{\dagger 2} \hat{\phi}_{\uparrow}^{2}+\hat{\phi}_{\downarrow}^{\dagger 2} \hat{\phi}_{\downarrow}^{2}\right)+\mathrm{g}_{\uparrow \downarrow} C \hat{\phi}_{\uparrow}^{\dagger} \hat{\phi}_{\downarrow}^{\dagger} \hat{\phi}_{\downarrow} \hat{\phi}_{\uparrow}\right\},
\end{aligned}
$$

where $\hat{\phi}=\left(\hat{\phi}_{\uparrow}, \hat{\phi}_{\downarrow}\right)^{T}$ and $C=\int_{0}^{\infty} f^{4}(r) r d r$. Since the total number of atoms is fixed, we neglect the term of $E_{0}$ in the following. We expand the field operator as

$$
\hat{\phi}_{\uparrow, \downarrow}(\theta)=\sum_{n} \hat{a}_{\uparrow, \downarrow n} \frac{e^{i n \theta}}{\sqrt{2 \pi}},
$$

where $\hat{a}_{\uparrow, \downarrow n}$ satisfies the bosonic commutation relations. Substituting Eq. (10) into the one-body part of Eq. (9), we obtain

$$
\begin{aligned}
\hat{\mathcal{H}}_{0}= & \sum_{n}\left[\frac{n^{2}}{2 R^{2}}\left(\hat{a}_{\uparrow n}^{\dagger} \hat{a}_{\uparrow n}+\hat{a}_{\downarrow n}^{\dagger} \hat{a}_{\downarrow n}\right)\right. \\
& \left.+\frac{i \kappa}{R} m\left(\hat{a}_{\downarrow n+1}^{\dagger} \hat{a}_{\uparrow n}-\hat{a}_{\uparrow n}^{\dagger} \hat{a}_{\downarrow n+1}\right)\right],
\end{aligned}
$$

where $m=n+1 / 2$, and we set $\Omega=0$ for simplicity. Using linear transformation as

$$
\begin{aligned}
& \hat{\alpha}_{m}^{-}=\hat{a}_{\uparrow n} \cos \alpha+i \hat{a}_{\downarrow n+1} \sin \alpha, \\
& \hat{\alpha}_{m}^{+}=\hat{a}_{\uparrow n} \sin \alpha-i \hat{a}_{\downarrow n+1} \cos \alpha,
\end{aligned}
$$

with $\tan 2 \alpha=2 \kappa R$, Eq. (11) is diagonalized as

$$
\hat{\mathcal{H}}_{0}=\sum_{m}\left(E_{m}^{+} \hat{\alpha}_{m}^{+\dagger} \hat{\alpha}_{m}^{+}+E_{m}^{-} \hat{\alpha}_{m}^{-\dagger} \hat{\alpha}_{m}^{-}\right)
$$

where

$$
E_{m}^{ \pm}=\frac{1}{2 R^{2}}\left(m^{2}+\frac{1}{4} \pm m \cos 2 \alpha\right) \pm \frac{\kappa}{R} m \sin 2 \alpha .
$$

These energies are two-fold degenerate because of $E_{m}^{-}=$ $E_{-m}^{+}$.

We consider the quantum many-body state that minimizes $\left\langle\hat{\mathcal{H}}_{0}\right\rangle$. The solution of $\partial E_{m}^{ \pm} / \partial m=0$ is given by $m=\mp \sqrt{1+(2 \kappa R)^{2}} / 2$. Let $\mp m_{0}$ be the half integers that are closest to $\mp \sqrt{1+(2 \kappa R)^{2}} / 2$ and minimize $E_{m}^{ \pm}$, which we define $E_{\min }=E_{\mp m_{0}}^{ \pm}$. The $N$-particle states that minimize $\left\langle\hat{\mathcal{H}}_{0}\right\rangle$ are then

$$
|N-p, p\rangle=\frac{\left(\hat{\alpha}_{-m_{0}}^{+\dagger}\right)^{N-p}\left(\hat{\alpha}_{m_{0}}^{-\dagger}\right)^{p}}{\sqrt{(N-p) !} \sqrt{p !}}|0\rangle,
$$

where $p=0,1, \cdots, N$. These states satisfy $\hat{\mathcal{H}}_{0} \mid N-$ $p, p\rangle=N E_{\min }|N-p, p\rangle$, and hence $(N+1)$-fold degenerate. When $\kappa R \gg 1$, we find $\alpha \simeq \pi / 4, m_{0} \simeq \kappa R$, and $E_{\min } \simeq-\kappa^{2} / 2$. In this case, the wave number along the ring is $\simeq m_{0} / R \simeq \kappa$. When $\kappa R<\sqrt{3} / 2$, we find $\alpha<\pi / 6$, $m_{0}=1 / 2$, and $n=0$, and the trivial state in which all the atoms are at rest is recovered. We therefore consider the case of $\kappa R>\sqrt{3} / 2$ in the following.

Next we examine the interaction energy. We assume that the interaction energy is much smaller than the kinetic and SOC energies, and that the ground state is spanned by the states in Eq. (15). The expectation value of the interaction part of Eq. (9) with respect to the many-body state in Eq. (15) is calculated to be

$$
\begin{aligned}
& \left\langle N-p, p\left|\hat{\mathcal{H}}_{\text {int }}\right| N-p, p\right\rangle \\
= & \frac{C}{16 \pi}\left[3 \mathrm{~g}+\mathrm{g}_{\uparrow \downarrow}+\left(\mathrm{g}-\mathrm{g}_{\uparrow \downarrow}\right) \cos 4 \alpha\right] N(N-1) \\
& -\frac{C}{8 \pi}\left(\mathrm{g}-\mathrm{g}_{\uparrow \downarrow}\right)(1+3 \cos 4 \alpha) p(N-p) .
\end{aligned}
$$

The matrix element $\left\langle N-p, p\left|\hat{\mathcal{H}}_{\text {int }}\right| N-p^{\prime}, p^{\prime}\right\rangle$ vanishes for $p \neq p^{\prime}$. In the last line of Eq. (16), $1+3 \cos 4 \alpha$ is negative. Therefore, the interaction energy is minimized by $p=0$ or $p=N$ for $\mathrm{g}>\mathrm{g}_{\uparrow \downarrow}$ and by $p=N / 2$ for $\mathrm{g}<\mathrm{g}_{\uparrow \downarrow}$, which correspond to the plane-wave and stripe phases, respectively. We note that the ground state for $\mathrm{g}<\mathrm{g}_{\uparrow \downarrow}$ is $|N / 2, N / 2\rangle$, which is the fragmented BEC [50].

\section{CONCLUSIONS}

We have investigated the effect of a toroidal trap on a SO coupled BECs with or without rotation. In contrast to the case of the harmonic trap, the interplay between the SOC and toroidal trap can result in a rich variety of ground and metastable states, such as triangular stripes, flower-petal patterns, and counter-circling states. We found that the condensate in a toroidal trap tends to have wave-number vectors in the azimuthal direction, which becomes more significant for a tighter toroidal trap. This finding can be explained by the Gaussian variational analysis. In the presence of rotation, the system is found to exhibit exotic ground-state vortex configurations by varying the strength of SOC and rotation frequency, and the number of vortices is the same as that in the momentum space. We have also formulated a quantum many-body problem for the quasi-1D ring trap for both miscible and immiscible cases. In the latter case, the ground state of the system is found to be a fragmented condensate. Owing to the recent developments in the experimental implementation of SOC and the high degrees of control over most of the system parameters, the various states found in this work may be observed in current experiments.

\section{Acknowledgments}

This work was supported by JSPS KAKENHI Grant Numbers JP16K05505, JP26400414, and JP25103007, by the NSFC under grants Nos. 61127901, and 11547126, by 
the key project fund of the CAS for the "Western Light" Talent Cultivation Plan under grant No. 2012ZD02, and by the Youth Innovation Promotion Association of CAS under grant No. 2015334.

X.-F. Zhang and M. Kato contributed equally to this work.

\section{Appendix: Variational analysis in the immiscible case}

Substituting Eq. (7) into the Gross-Pitaevskii energy functional and integrating with respect to $x$, we obtain

$$
\begin{aligned}
E_{\text {kinetic }}= & \frac{1}{4 \sigma^{2}}+\frac{k^{2}}{2}, \\
E_{\text {potential }}= & \frac{\sigma^{2}}{4}+\frac{X^{2}}{2}, \\
E_{\mathrm{soc}}= & -\kappa\left(k+\frac{k_{y}}{k} \frac{X}{\sigma^{2}}\right) e^{-\frac{X^{2}}{\sigma^{2}}} \\
& +i e^{-k_{x}^{2} \sigma^{2}} k_{y} \cos \left(2 k_{x} X+\theta\right) \cos 2 k_{y} y, \\
E_{\text {interaction }}= & \frac{1}{8 \sqrt{2 \pi} \sigma}\left[g\left(1+2 e^{-\frac{2 X^{2}}{\sigma^{2}}}\right)+g_{\uparrow \downarrow}\right. \\
& \left.+\left(g_{\uparrow \downarrow}-g\right) e^{-2 k_{x}^{2} \sigma^{2}-\frac{2 X^{2}}{\sigma^{2}}} \cos 4 k_{y} y\right],(\mathrm{A}
\end{aligned}
$$

where $E_{\text {kinetic }}$ and $E_{\text {potential }}$ are the same as those in the miscible case in Eq. (6). The second line of Eq. (A.3) vanishes by integrating with respect to $y$. Similarly, the second line of Eq. (A.4) also vanishes for $k_{y} \neq 0$. In this case, assuming $X \ll \sigma$, one finds that $k_{x}=0, k_{y} \simeq$ $\pm \kappa$, and $X \simeq \pm \kappa / \sigma^{2}$ minimize the total energy. The energy lowered by the shift $X$ is $\simeq \kappa^{2} /\left(2 \sigma^{4}\right)$. When the immiscible interaction energy dominates the soc energy, the second line of Eq. A.4 is minimized by $k_{x}=k_{y}=0$ and $X=0$, which corresponds to the state shown in Fig. 1(a).
[1] Y.-J. Lin, R. L. Compton, K. Jiménez-García, J. V. Porto, and I. B. Spielman, Nature (London) 462, 628 (2009).

[2] Y.-J. Lin, R. L. Compton, A. R. Perry, W. D. Phillips, J. V. Porto, and I. B. Spielman, Phys. Rev. Lett. 102, 130401 (2009).

[3] Y.-J. Lin, K. Jiménez-García, and I. B. Spielman, Nature (London) 471, 83 (2011).

[4] Y.-J. Lin, R. L. Compton, K. Jiménez-García, W. D. Phillips, J. V. Porto, and I. B. Spielman, Nat. Phys. 7, 531 (2011).

[5] P. Wang, Z.-Q. Yu, Z. Fu, J. Miao, L. Huang, S. Chai, H. Zhai, and J. Zhang, Phys. Rev. Lett. 109, 095301 (2012).

[6] B. M. Anderson, G. Juzeliūnas, V. M. Galitski, and I. B. Spielman, Phys. Rev. Lett. 108, 235301 (2012).

[7] Z. Fu, L. Huang, Z. Meng, P. Wang, L. Zhang, S. Zhang, H. Zhai, P. Zhang, and J. Zhang, Nat. Phys. 10, 110 (2014).

[8] S. C. Ji, J. Y. Zhang, L. Zhang, Z. D. Du, W. Zheng, Y. J. Deng, H. Zhai, S. Chen, and J. W. Pan, Nat. Phys. 10, 314 (2014).

[9] L. Huang, Z. Meng, P. Wang, P. Peng, S.-L. Zhang, L. Chen, D. Li, Q. Zhou, and J. Zhang, Nat. Phys. 12, 540 (2016).

[10] Z. Wu, L. Zhang, W. Sun, X.-T. Xu, B.-Z. Wang, S.-C. Ji, Y. Deng, S. Chen, X.-J Liu, J.-W. Pan, Science 354, 83 (2016).

[11] M. Z. Hasan and C. L. Kane, Rev. Mod. Phys. 82, 3045 (2010).

[12] X.-L. Qi and S.-C. Zhang, Rev. Mod. Phys. 83, 1057
(2011).

[13] J. Dalibard, F. Gerbier, Juzeliūnas, and P. Öhberg, Rev. Mod. Phys. 83, 1523 (2011).

[14] C. J. Wu, I. Mondragon-Shem, and X. F. Zhou, Chin. Phys. Lett. 28, 097102 (2011).

[15] N. Goldman, G. Juzeliūnas, P. Öhberg, and I. B. Spielman, Rep. Prog. Phys. 77, 126401 (2014).

[16] H. Zhai, Rep. Prog. Phys. 78, 026001 (2015).

[17] K. Sun, C. Qu, and C. Zhang, Phys. Rev. A 91, 063627 (2015).

[18] M. Demarco and H. Pu, Phys. Rev. A 91, 033630 (2015).

[19] C. Qu, K. Sun, and C. Zhang, Phys. Rev. A 91, 053630 (2015).

[20] C. Wang, C. Gao, C.-M. Jian, and H. Zhai, Phys. Rev. Lett. 105, 160403 (2010).

[21] T.-L. Ho and S. Zhang, Phys. Rev. Lett. 107, 150403 (2011).

[22] S. Sinha, R. Nath, and L. Santos, Phys. Rev. Lett. 107, 270401 (2011).

[23] H. Hu, B. Ramachandhran, H. Pu, and X.-J. Liu, Phys. Rev. Lett. 108, 010402 (2012).

[24] Y. Zhang, L. Mao, and C. Zhang, Phys. Rev. Lett. 108, 035302 (2012).

[25] Y. Li, L. P. Pitaevskii, and S. Stringari, Phys. Rev. Lett. 108, 225301 (2012).

[26] T. Kawakami, T. Mizushima, M. Nitta, and K. Machida, Phys. Rev. Lett. 109, 015301 (2012).

[27] B. Ramachandhran, B. Opanchuk, X.-J. Liu, H. Pu, P. D. Drummond, and H. Hu, Phys. Rev. A 85, 023606 (2012). 
[28] E. Ruokokoski, J. A. M. Huhtamäki, and M. Möttönen, Phys. Rev. A 86, 051607(R) (2012).

[29] X.-Q. Xu and J. H. Han, Phys. Rev. Lett. 107, 200401 (2011).

[30] J. Radić, T. A. Sedrakyan, I. B. Spielman, and V. Galitski, Phys. Rev. A 84, 063604 (2011).

[31] X. F. Zhou, J. Zhou, and C. J. Wu, Phys. Rev. A 84, 063624 (2011).

[32] A. Aftalion and P. Mason, Phys. Rev. A 88, 023610 (2013).

[33] H. Sakaguchi and K. Umeda, J. Phys. Soc. Jpn. 85, 064402 (2016).

[34] S. Eckel, J. G. Lee, F. Jendrzejewski, N. Murray, C. W. Clark, C. J. Lobb, W. D. Phillips, M. Edwards, and G. K. Campbell, Nature (London) 506, 200 (2014).

[35] A. A. Wood, B. H. J. McKellar, and A. M. Martin, Phys. Rev. Lett. 116, 250403 (2016).

[36] F. Jendrzejewski, S. Eckel, N. Murray, C. Lanier, M. Edwards, C. J. Lobb, and G. K. Campbell, Phys. Rev. Lett. 113, 045305 (2014).

[37] L. Corman, L. Chomaz, T. Bienaimé, R. Desbuquois, C. Weitenberg, S. Nascimbéne, J. Dalibard, and J. Beugnon, Phys. Rev. Lett. 113, 135302 (2014).

[38] S. Beattie, S. Moulder, R. J. Fletcher, and Z. Hadzibabic, Phys. Rev. Lett. 110, 025301 (2013).
[39] K. C. Wright, R. B. Blakestad, C. J. Lobb, W. D. Phillips, and G. K. Campbell, Phys. Rev. Lett. 110, 025302 (2013).

[40] C. Ryu, P. W. Blackburn, A. A. Blinova, and M. G. Boshier, Phys. Rev. Lett. 111, 205301 (2013).

[41] C. Ryu, M. F. Andersen, P. Cladé, V. Natarajan, K. Helmerson, and W. D. Phillips, Phys. Rev. Lett. 99, 260401 (2007).

[42] S. Bargi, F. Malet, G. M. Kavoulakis, and S. M. Reimann, Phys. Rev. A 82, 043631 (2010).

[43] M. Abad, A. Sartori, S. Finazzi, and A. Recati, Phys. Rev. A 89, 053602 (2014).

[44] A. M. Mateo, A. Gallemí, M. Guilleumas, and R. Mayol, Phys. Rev. A 91, 063625 (2015).

[45] R. Kanamoto, H. Saito, and M. Ueda, Phys. Rev. Lett. 94, 090404 (2005).

[46] T. Shimodaira, T. Kishimoto, and H. Saito, Phys. Rev. A 82, 013647 (2010).

[47] A. Aftalion and P. Mason, Phys. Rev. A 81, 023607 (2010).

[48] M. Abad, Phys. Rev. A 93, 033603 (2016).

[49] A. L. Fetter, Phys. Rev. A 89, 023629 (2014).

[50] S.-W. Song, Y.-C. Zhang, H. Zhao, X. Wang, and W.-M. Liu, Phys. Rev. A 89, 063613 (2014). 